\title{
Post-AGB stars in the LMC and SMC
}

\author{
Hans Van Winckel ${ }^{1}$, Els van Aarle ${ }^{2}$, Peter R. Wood ${ }^{2}$, \\ Devika Kamath ${ }^{2}$, Tom Lloyd Evans ${ }^{3}$, Kenneth De Smedt ${ }^{1}$, \\ Nadya Gorlova ${ }^{1}$, and Clio Gielen ${ }^{1}$ \\ ${ }^{1}$ Instituut voor Sterrenkunde, K.U.Leuven, \\ Celestijnenlaan 200D, 3001 Leuven, Belgium, \\ email: Hans.VanWinckel@ster .kuleuven. be \\ ${ }^{2}$ Research School of Astronomy and Astrophysics, Australian National University \\ ${ }^{3}$ SUPA, School of Physics \& Astronomy, University of St Andrews, North Haugh, St Andrews \\ KY16 9SS, Scotland, UK
}

\begin{abstract}
Galactic post-AGB stars show a large diversity in chemical compositions as well as in the kinematical and geometrical properties of their circumstellar material. The theoretical interpretation is hampered by the lack of good distances to almost all Galactic objects. We therefore initiated a large project to study systematically post-AGB stars in the Large and Small Magellanic Cloud (LMC, SMC respectively). Here we report on our preliminary results.
\end{abstract}

Keywords. Stars: AGB and post-AGB, stars: evolution, Magellanic Clouds, circumstellar matter

\section{Introduction}

The final evolution of low- and intermediate-mass stars is a rapid transition from the Asymptotic Giant Branch (AGB) over the post-AGB transit towards the Planetary Nebula Phase (PN), before the stellar remnant cools down as a White Dwarf (WD). During this evolution the central star changes from a dust embedded cool AGB star with a radius $\sim 1 \mathrm{AU}$ to a WD the size of the earth. Although this evolutionary scheme may be generally acknowledged (Vassiliadis \& Wood 1994; Blöcker 1995) there is no understanding from first principles of different important physical processes that govern these evolutionary phases. As the evolution is fast, not many Galactic post-AGB stars are known and a database of Galactic post-AGB stars was presented by Szczerba (these proceedings. See also http://www.ncac.torun.pl/postagb2).

During the late AGB evolution the 3rd dredge-up phenomenon is active, during which products of the internal nucleosynthesis are brought to the surface of the star. This process leaves behind a poorly-known ${ }^{13} \mathrm{C}$ pocket in which the synthesis of heavy elements by slow neutron capture (s-process) subsequently occurs deep in the stellar interior. Synthesis by the s-process, is an important contributor to the cosmic abundances past the iron peak. Additionally, AGB stars are thought to be very important contributors to the total carbon and nitrogen enrichment of the parent galaxy. Post-AGB photospheres bear witness to the total AGB chemical changes. Their properties make that the spectra are dominated by atomic transitions which allow an abundance determination of a very wide range of chemical species, from $\mathrm{CNO}$ to elements past the Ba peak.

The chemical diversity of post-AGB stars is large and versatile (Sect.2), however, and not all surface abundance patterns can be understood by an accumulation of internal enrichment processes. The poorly known distances and hence luminosities and masses of the limited Galactic post-AGB sample, hamper the interpretation of the variety of abundances in the broader theoretical context of stellar (chemical) evolution. We therefore 
turned to samples with well constrained distances and in this contribution we focus on the post-AGB stars of the LMC and SMC (Sect. 3).

\section{Galactic optically bright Post-AGB stars}

Specific Galactic post-AGB stars are associated with circumstellar material displaying the $21 \mu \mathrm{m}$ dust feature. This feature remains unidentified but it is only seen in circumstellar material around carbon-rich post-AGB stars and it is often in associations with PAH emission bands. These post-AGB stars are indeed the most s-process enriched objects known to date, while other post-AGB stars are not enriched at all (e.g Van Winckel 2003).

A very particular example of photospheric chemical anomaly is called depletion, in which the stellar photosphere displays abundance patterns resembling the gas-phase of the interstellar medium (ISM): refractory elements like Ca, and Fe are depleted with respect to elements with a low dust condensation temperature like S and Zn (e.g. Sumangala Rao et al. 2011, and references therein). In almost all cases the SEDs of the depleted objects are distinct. The SED is bimodal (Fig. 1), with peaks in the visible and mid-IR, including a prominent near-IR excess, which in interpreted as coming from hot dust in a stable dusty disc (e.g. Van Winckel 2003, and references therein). The characteristic SED was used to start systematic searches for such Galactic systems and our original list of

De Ruyter et al. (2006) is ever expanding and contains now about 80 objects. The discs are very compact (e.g. Deroo et al. 2007). The high degree of dust processing (e.g. Gielen et al. 2011) points to a long-lived structure.

These disc systems are associated with binaries (e.g. Van Winckel et al. 2009) which avoided spiral-in during their evolution. Typical periods are one to several years and the orbits are remarkably eccentric. Not all disc objects are depleted, however, and not all depleted stars have still an infrared excess. This may very well be an indication that the disc evolution occurs at timescales similar or shorter than the evolution of the central star in its crossing of the HR-diagram.

The orbits are too small to accommodate a AGB star (Van Winckel et al. 2009) and we use our new high-resolution spectrograph HERMES (Raskin et al. 2011) to obtain time series of high-quality spectra which cover the orbital phases. A first detailed analyses is presented by us (Gorlova et al. 2011) where it is shown that BD+46.442 is a evolved star in a binary surrounded by a dusty disc. The phase-dependent line-profiles of $\mathrm{H}_{-} \alpha$ show that the companion is likely surrounded by a gas accretion disc in which a jet is launched with very high speed ( $>400 \mathrm{~km} / \mathrm{s}$ ). This illustrates the fact that disc sources are interacting binaries in which high velocity jets can be created around the companions via an accretion disc (see Franck and Riera these proceedings) in addition to the larger circumbinary dusty
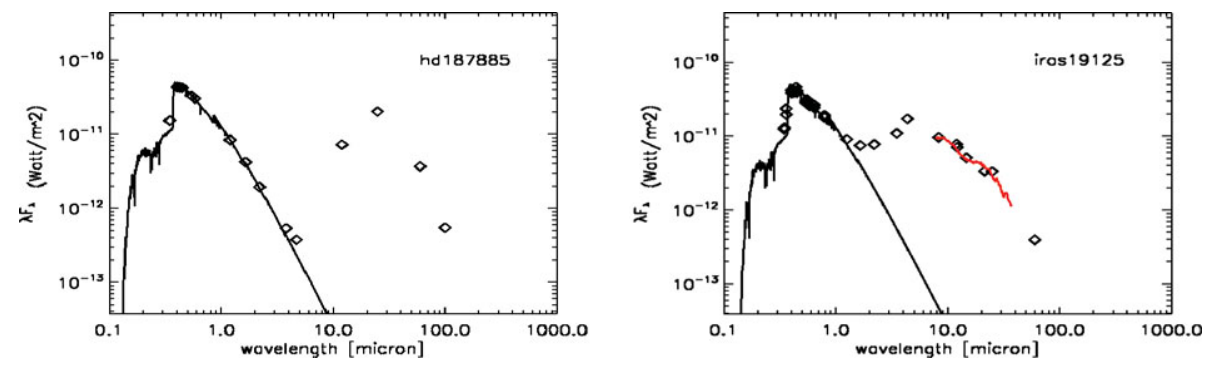

Figure 1. We devide the SEDs of visually bright post-AGB stars fall into two categories: left an object with a double-peaked SED indicative of a cooling and expaning former AGB wind (example shown is HD 187885). right: and SED typical of a disc (example shown is IRAS19125+0343). 
disc. The disc sources form ideal objects to study binary interaction processes but also here, the poorly known distances hamper the full theoretical interpretation.

\section{LMC and SMC samples}

Systematic searches of the rare post-AGB stars in external galaxies only became possible after the release of deep infrared surveys. We exploited the release of the infrared LMC SAGE-Spitzer survey (Meixner et al. 2006) and searched for luminous, optically bright stars with infrared colours indicative of a past history of heavy dusty mass loss, be it in a disc or an expanding outflow. Our final result consists of some 1400 good candidate post-AGB stars in the LMC alone of which 70 are spectroscopically confirmed (van Aarle et al. 2011). The sample selection of the SMC was based on the similar grounds using the SAGE-SMC survey (Gordon et al. 2011) and resulted in some 400 candidates (Kamath et al. 2011).

It is remarkable that about half of these sources display SEDs indicative of discs (van Aarle et al. 2011) and, similar to what is observed in our Galaxy, they are likely evolved binaries! The spectral confirmation of all these candidates is in full progression by using the $2.3 \mathrm{~m}$ Anglo-Australian Telescope (Kamath et al. 2011) equipped with the AAOmega spectrograph. Soon, we will have a very complete census of spectroscopically characterised post-AGB objects in both the LMC and SMC, and this for disc sources as well as expanding shell sources. This sample will provide very good constraints on both the final evolution of single as well as binary stars.

In a pilot program, we were granted time on the $8.2 \mathrm{~m}$ VLT to obtain high-resolution spectra on a restricted sample. Coupled to computation of detailed model atmospheres, these spectra allow us to determine the chemical composition of the stellar surface layers. It turned out to be a very successful first step towards the study of extra-galactic post-AGB objects. MACHO47.2496.8, e.g. is strongly enriched in carbon and s-process elements (Reyniers et al. 2007b) The metallicity of $[\mathrm{Fe} / \mathrm{H}]=-1.4$ is surprisingly low for a field LMC star. The $\mathrm{C} / \mathrm{O}$ ratio is above 2, and the s-process enrichment is very large: the light s-process elements are enhanced by 1.2 dex compared to iron $([\mathrm{ls} / \mathrm{Fe}]=+1.2)$, while for the heavy s-process elements, an even stronger enrichment is measured: $[\mathrm{hs} / \mathrm{Fe}]=+2.1$.

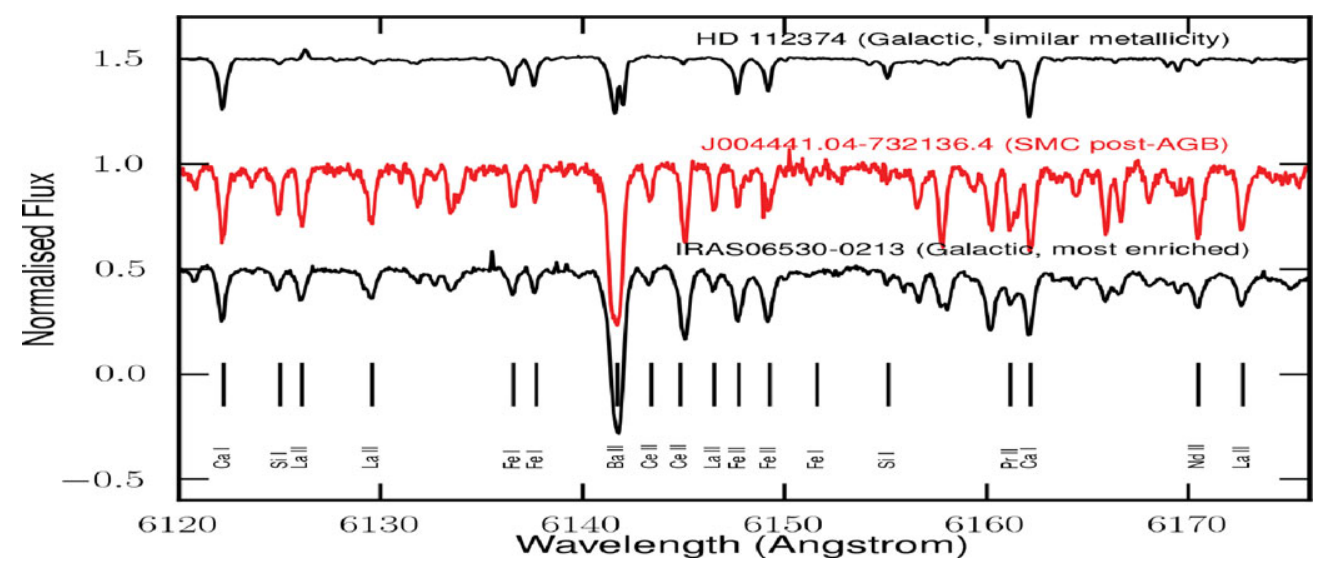

Figure 2. Illustration of the rich spectrum of J004441.04-732136.3, a $21 \mu \mathrm{m}$ source of the SMC. Spectral comparison stars are HD 112374 (top, non s-process enriched) and IRAS06530-0213 (s-process enriched). These are Galactic post-AGB stars of similar temperature and mealliticty. The s-process enrichement is so large that lines from s-process elements dominate the spectrum (De Smedt et al. 2012). 
The s-process abundance patterns can only be understood assuming a surprisingly low efficiency of the ${ }^{13} \mathrm{C}$ pocket (Bonačić Marinović et al. 2007).

In Fig. 2 below, we show a spectrum of the SMC source J004441.04-732136.3, which is the only source in the SMC known to display a $21 \mu \mathrm{m}$ feature (Volk et al. 2011). Like in the Galaxy, these objects are post carbon stars and this source is one of the most s-process enriched objects known to date (De Smedt et al. 2012) !

Similar to what is detected in the Galaxy, also in the LMC, depleted photospheres seem to be common. In all cases depletion abundance patterns are associated with disc sources. These disc sources cover a whole luminosity range in the LMC (van Aarle et al. 2011) but the determination of orbital elements will be a serious observational challenge.

In the next phase of our endeavors, the obtained abundance patterns of shell sources will be confronted with tailored state-of-the-art chemical AGB models appropriate for the LMC and SMC. The luminosity of post-AGB stars in the Clouds can be directly converted into an initial mass range via AGB evolution calculations, although some uncertainty arises from uncertainties in the adopted mass loss rate as well as on the determination of the total line-of-sight reddening. The confrontation between the observed abundance patterns, the metallicity, the luminosity range and the theoretical predictions, will bring direct and stringent constraints on the parameters governing the mixing in AGB stars as well as the nucleosynthesis induced by the thermal pulses on the AGB. This will allow to probe directly hitherto poorly constrained parameters like the 3rd dredge-up occurrence, the s-process efficiency (the abundance distribution as well as the global enrichment) as functions of luminosity and initial metallicity. Only a combination of high-quality data of a well defined sample of post-AGB stars in the Magellanic Clouds with a range of luminosities, and a detailed theoretical modelling, can imply significant progress in our understanding of the dredge-up processes and the associated s-process nucleosynthesis. This is the ultimate goal of our research.

\section{References}

Blöcker, T. 1995, A\&A, 299, 755

Bonačić Marinović, A., Lugaro, M., Reyniers, M., \& Van Winckel, H. 2007, A\&AA, 472, L1

De Ruyter, S., Van Winckel, H., Maas, T., et al. 2006, A\&GA, 448, 641

Deroo, P., Acke, B., Verhoelst, T., et al. 2007, A\&A, 474, L45

De Smedt, K., Van Winckel H., Wood P. R., et al. 2012, A\&A, in prep

Gielen, C., Van Winckel, H., Reyniers, M. et al. 2009, A\& A, 508, 1391

Gielen, C., Bouwman, J., van Winckel, H., et al. 2011, A\&AA, 533, A99+

Gorlova, N., Van Winckel, H., Gielen, C., et al. 2011, A\& $A$, submitted

Gordon, K. D., Meixner, M., Meade, M. R., et al. 2011, AJ, 142, 102

Kamath, D., Wood, P., Van Winckel, H., \& van Aarle, E. 2011, in 9th Pacific Rim Conference on Stellar Astrophysics, ed., ASP conference series, in press

Kwok, S., Volk, K. M., \& Hrivnak, B. J. 1989, ApJ, 345, L51

Meixner, M., Gordon, K. D., Indebetouw, R., et al. 2006, AJ, 132, 2268

Sumangala Rao, S., Giridhar, S., \& Lambert, D. L., 2011, ApJ, in press (astro-ph 1109.1676)

Raskin, G., Van Winckel, H., Hensberge, H., et al., 2011, A\&SA, 526, 69

Reyniers, M. \& Van Winckel, H., 2007a, A\&SA, 463 , 1

Reyniers, M., Abia, C., Van Winckel, H., et al. 2007b, A\&A, 461, 641

van Aarle, E., Van Winckel, H., Lloyd Evans, T., et al. 2011, A\&GA, 530, A90+

Van Winckel, H. 2003, ARAA, 41, 391

Van Winckel, H., Lloyd Evans, T., Briquet, M., et al. 2009, A\&A, 505, 1221

Vassiliadis, E. \& Wood, P. R. 1994,ApJS , 92, 125

Volk, K., Hrivnak, B. J., Matsuura, M., et al. 2011, ApJ, 735, 127 